\title{
Core-equivalence for the Nash bargaining solution
}

\author{
Walter Trockel* \\ Institute of Mathematical Economics (IMW), Bielefeld University** and \\ W.P. Carey School of Business, Arizona State University, Tempe AZ
}

November 2003

\begin{abstract}
Core equivalence and shrinking of the core results are well known for economies. The present paper establishes counterparts for bargaining economies, a specific class of production economies (finite and infinite) representing standard two-person bargaining games and their continuum counterparts as coalition production economies. Thereby we get core equivalence of the Nash solution. The results reconfirm the Walrasian approach to Nash bargaining of Trockel (1996). Moreover we establish the same speed of convergence as is known from Debreu (1975) and Grodal (1975) for replicated pure exchange economies and for regular purely competitive sequences of economies, respectively.
\end{abstract}

* This article is dedicated to Birgit Grodal, a friend since 30 years. Financial support of the DFG under grant \#444 USA 111/2/03 is gratefully acknowledged.

** Address: Postfach 100131, D-33501 Bielefeld email: WTrockel@wiwi.uni-bielefeld.de 


\section{Introduction}

The Nash solution of two person bargaining games introduced by Nash (1953) has been characterized in manifold ways. Some of them hint to the fact that it reflects some kind of immunity against strategic exploitation. So does Rubinstein's (1982) alternating offer game whose subgame perfect equilibrium approaches the Nash solution the closer the less the discounting of future is by the strength of alternative future options. Also Trockel's (1996) Walrasian characterization of the Nash solution reflects the pressure of competition providing sufficient outside options. In the latter approach, following ideas of Shapley (1969), the two players' utilities are treated as the two commodities in an artificial economy with production and private property.

The Walrasian characterization establishes the vector $\lambda$ in Shapley's $\lambda$-transfer value as a competitive price system thereby proving a conjecture of Shubik (1985).

In the present paper we relate the Nash solution with the Edgeworthian rather than the Walrasian version of perfect competition. To do so, we define an artificial coalition production economy (cf. Hildenbrand (1974)) representing a two person bargaining game.

In a similar way the Nash solution has been applied in Mayberry et al. (1953) to define a specific solution for a duopoly situation and comparing it with other solutions, among them the Edgeworth contract curve. The relation between these two solutions will be the object of our investigation in this paper.

Though it would not be necessary to be so restrictive we define a two person bargaining game as the closed subgraph of a continuously differentiable strictly decreasing concave function $f:[0,1] \longrightarrow[0,1]$ with $f(0)=1$ and $f(1)=0$.

$S:=$ subgraphf $:=\left\{\left(x_{1}, x_{2}\right) \in[0,1]^{2} \mid x_{2} \leq f\left(x_{1}\right)\right\}$

The normalization reflects the fact that bargaining games are usually considered to be given only up to positive affine transformations. Smoothness makes life easier by admitting unique tangents.

The model $S$ is general enough for our purpose of representation by a coalition production economy. In particular, $S$ is the intersection of some strictly convex comprehensive set with the positive orthant of $\mathbb{R}^{2}$.

\section{The basic model}

Define for any $S$ as described in section 1 a two person coalition production economy $\mathcal{E}^{S}$ as follows: 
$\mathcal{E}^{S}:=\left(\left(e_{i}, \succsim_{i}, Y_{i}\right)_{i=1,2},\left(\vartheta_{i j}\right)_{i, j=1,2}\right)$ such that

$e_{i}=(0,0), x=\left(x_{1}, x_{2}\right) \succsim_{i} x^{\prime}=\left(x_{1}^{\prime}, x_{2}^{\prime}\right) \Leftrightarrow x_{i} \geq x_{i}^{\prime}, i=1,2$

$\vartheta_{11}=\vartheta_{22}=1, \vartheta_{12}=\vartheta_{21}=0, Y_{1}=Y_{2}=\left(\frac{1}{2}\right) S$

The zero initial endowments reflect the idea that all available income in this economy comes from shares in production profits.

Each agent owns fully a production possibility set that is able to produce for any $x \in S$ the bundle $\left(\frac{1}{2}\right) x$ without any input.

Both agents are interested in only one of the two goods called "agent $i^{\prime} s$ utility", $i=1,2$.

Without any exchange agent $i$ would maximize his preference by producing and consuming one half unit of commodity $i$ and zero units of commodity $3-i, i=1,2$.

However, the agents would recognize immediately that they left some joint utility unused on the table.

Given exchange possibilities for the two commodities they would see that improvement would require exchange or, to put it differently, coordinated production (see Figure 1).

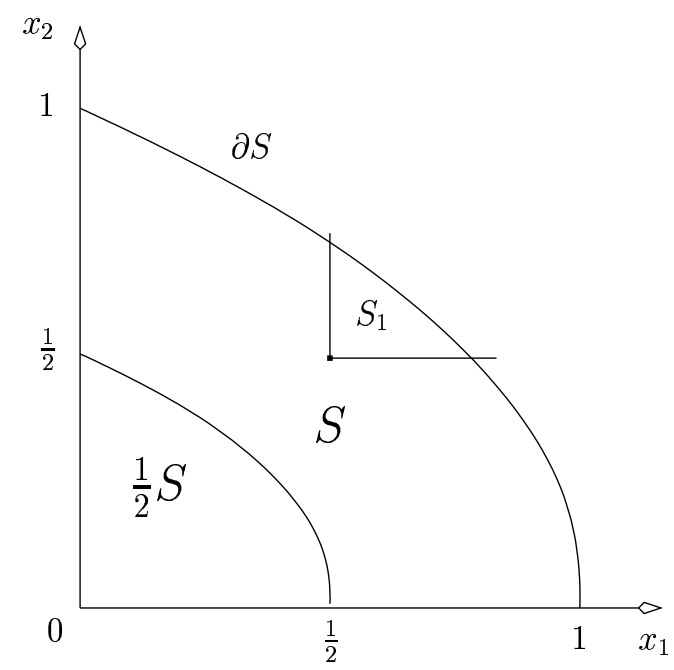

Figure 1

The point $\left(\frac{1}{2}, \frac{1}{2}\right)$ corresponds to the vector of initial endowments, the set $S_{1}:=S \cap$ $\left(\left\{\left(\frac{1}{2}, \frac{1}{2}\right)\right\}+\mathbb{R}_{+}^{2}\right)$ to the famous lens and the intersection of $S_{1}$ with the efficient boundary of $S$, i.e. $S_{1} \cap \partial S$, to the core in the Edgeworth Box. This is exactly what Mayberry and al. (1953, p. 144) call the Edgeworth contract curve in their similar setting. 
The according notions of improvement and of the core are analogous to the ones used for Coalitional Production Economies by Hildenbrand (1974, p. 211).

$\tilde{Y}:\{\{1\},\{2\},\{1,2\}\} \Longrightarrow \mathbb{R}^{2}$ with $\tilde{Y}(\{1\})=Y_{1}, \tilde{Y}(\{2\})=Y_{2}, \tilde{Y}(\{1,2\})=S$, is the production correspondence, which is additive, as $Y(\{1\} \cup\{2\})=Y_{1}+Y_{2}=S$.

An allocation $x^{i}=\left(\left(x_{1}^{i}, x_{2}^{i}\right)\right)_{i=1,2}$ for $\mathcal{E}^{S}$ is $T$-attainable for $T \in\{\{1\},\{2\},\{1,2\}\}$ if

$\sum_{i \in T} x^{i} \in \tilde{Y}(T)$; it is called attainable if it is $\{1,2\}$-attainable.

An allocation $\left(x^{1}, x^{2}\right)$ can be improved upon by a coalition $T \in\{\{1\},\{2\},\{1,2\}\}$ if there is a $T$-attainable allocation $\left(y^{1}, y^{2}\right)$ such that $\forall i \in T: y^{i} \succ_{i} x^{i}$.

The core of $\mathcal{E}^{S}$ is the set of $\{1,2\}$-attainable allocations that cannot be improved upon.

The analogous definitions hold for all $n$-replicas $\mathcal{E}_{n}^{S}$ of $\mathcal{E}^{S}, n \in \mathbb{N}$.

Notice that our choice of $Y_{i}=\left(\frac{1}{2}\right) S, i=1,2$ ensures the utility allocation $\left(\frac{1}{2}, \frac{1}{2}\right)$ for the two players in case of non-agreement. This differs from Nash's status quo or threat point $(0,0)$.

Formalizing an $n$-replica economy $\mathcal{E}_{n}^{S}$ is standard. All characteristics are replaced by $n$ tupels of identical copies of these characteristics. In particular $\mathcal{E}_{n}^{S}$ has $2 n$ agents, $n$ of each of the two types 1 and 2 . And the total production possibility set for the grand coalition of all $2 n$ agents is $n S$.

Although the use of strict convex preferences as in Debreu and Scarf (1963) is not available here a short moment of reflection shows that a major part of their arguments can be used in our case as well.

In the case of a continuum of agents a coalition production economy representing the bargaining game $S$ is a map

$\tilde{\mathcal{E}}^{S}:([0,1], \mathcal{B}[0,1], \lambda) \longrightarrow 2^{\mathbb{R}_{+}^{2}} \times \mathbb{R}_{+}^{2} \times C^{\circ}(S) \times\{0,1\}^{[0,1]}: t \mapsto\left(\tilde{Y}(t), e_{t}, u_{t}, \vartheta_{t}\right)$,

with $\tilde{Y}(t):=S, e_{t}=(0,0), u_{t}:=1_{\left[0, \frac{1}{2}\right]}(t) \cdot \operatorname{proj}_{1}+1_{\left.] \frac{1}{2}, 1\right]}(t) \cdot \operatorname{proj}_{2}$

for all $t \in[0,1]$. For each $t$ the share function $\vartheta_{t}$ is given by $\vartheta_{t}:[0,1] \longrightarrow\{0,1\}: s \mapsto$ $\vartheta_{t}(s):=\delta_{t, s}($ Kronecker delta $)$.

An allocation $\tilde{x}:[0,1] \longrightarrow[0,1]^{2}$ is T-attainable for $T \in \mathcal{B}[0,1]$ if $\int \tilde{x}(t) \lambda(d t) \in \int_{T} \tilde{Y}(t) \lambda(d t)=$ $\lambda(T) S$.

A [0,1]-attainable allocation $\tilde{x}$ can be improved upon by a coalition $T \subset \mathcal{B}[0,1]$ via a 
T-attainable allocation $\tilde{y}$, if $\lambda$ a.e. in $T: u_{t}(\tilde{y}(t))>u_{t}(\tilde{x}(t))$. The core of $\mathcal{E}^{S}$ is the set of attainable allocations that cannot be improved upon.

\section{Core equivalence}

Consider $S$ as illustrated in Figure 2.

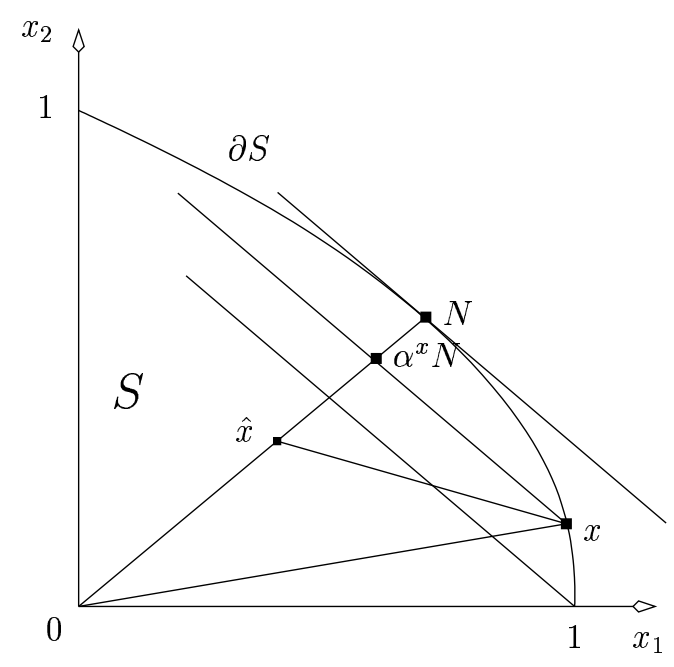

Figure 2

It is well known that the vector $\left(N_{2}, N_{1}\right)$ is normal to $\partial S$ at the Nash solution $N$ of $S$. For any $x \in \partial S$ with $x_{1}>N_{1}$, and thus $x_{2}<N_{2}$ we have:

$\left(N_{2}, N_{1}\right) \cdot\left(x_{1}, x_{2}\right) \in\left[\left(N_{2}, N_{1}\right) \cdot(1,0),\left(N_{2}, N_{1}\right) \cdot\left(N_{1}, N_{2}\right)\right]=\left[N_{2}, 2 N_{1} N_{2}\right]$.

Therefore we get

$\left(N_{2}, N_{1}\right) \cdot\left(x_{1}, x_{2}\right)=\left(N_{2}, N_{1}\right) \cdot\left(\alpha^{x} N_{1}, \alpha^{x} N_{2}\right)=2 \alpha^{x} N_{1} N_{2}$ for some $\alpha^{x} \in\left[\frac{1}{2 N_{1}}, 1\right]$.

The efficient point $x$ can only be realized by the grand coalition $[0,1]$ if $\lambda$ a. e. agent produces $x$. Clearly, $x$ is allocated to the agents in such a way that each agent in $\left[0, \frac{1}{2}\right]$ receives $\left(2 x_{1}, 0\right)$ and each agent in $\left.] \frac{1}{2}, 1\right]$ receives $\left(0,2 x_{2}\right)$. The idea for the construction of a coalition $C^{x}$ that improves upon $x$ is as follows. Let all members of $C^{x}$ produce some alternative point more favorable for type 2 agents, hence less favorable for type 1 agents. But choose the set $C_{1}^{x}$ of type 1 agents in $C^{x}$ so small that by allocating among them equally the whole production of good 1 each of them is even better off than before.

The type 2 agents improve by choosing that alternative point in such a way that the increase in production of good 2 per capita overcompensates the loss caused by the fact 
that only few type 1 agents are contributing to the production of good 2 .

First we choose $\hat{x}:=x+\beta^{x}\left(-x_{1}, x_{2}\right)$ in such a way that it is in the segment $[0, N]$. As $\left(-N_{1}, N_{2}\right)$ is steeper than $\left(-x_{1}, x_{2}\right)$ we know that $\left.\hat{x} \in\right] 0, \alpha^{x} N[$.

Now choose a small set $C_{1}^{x}$ of type 1 agents of measure $\alpha_{1}^{x}>0$ and a set $C_{2}^{x}$ of type 2 agents of measure $\alpha_{2}^{x}>\alpha_{1}^{x}$ with $\alpha_{2}^{x}<\frac{1}{2}$.

Denote the union of these two sets $C^{x}$. So $C^{x}$ has the measure $\alpha_{1}^{x}+\alpha_{2}^{x}$. Let each of the members of $C^{x}$ produce $\hat{x}:=\left(\left(1-\beta^{x}\right) x_{1},\left(1+\beta^{x}\right) x_{2}\right)$.

Their total production is $\left(\alpha_{1}^{x}+\alpha_{2}^{x}\right)\left(\left(1-\beta^{x}\right) x_{1},\left(1+\beta^{x}\right) x_{2}\right)$.

We want to reallocate this by distributing equally the total amount of good $i$ among type $i$ agents, $i=1,2$ in such a way that each agent gets exactly what he received from $x$. Hence we must have:

$\int_{C^{x}}\left(\left(1-\beta^{x}\right) x_{1},\left(1+\beta^{x}\right) x_{2}\right) d \lambda=\left(\alpha_{1}^{x}+\alpha_{2}^{x}\right)\left(\left(1-\beta^{x}\right) x_{1},\left(1+\beta^{x}\right) x_{2}\right)=\left(\begin{array}{lll}\alpha_{1}^{x} & \left.2 x_{1}, \alpha_{2}^{x} 2 x_{2}\right)\end{array}\right.$ $=\int_{C_{1}^{x}}\left(2 x_{1}, 0\right) d \lambda+\int_{C_{2}^{x}}\left(0,2 x_{2}\right) d \lambda$.

Therefore:

$\left(\alpha_{1}^{x}+\alpha_{2}^{x}\right)\left(1-\beta^{x}\right)=2 \alpha_{1}^{x}$ and $\left(\alpha_{1}^{x}+\alpha_{2}^{x}\right)\left(1+\beta^{x}\right)=2 \alpha_{2}^{x}$ hence:

$\left.\beta^{x}=\frac{\alpha_{2}^{x}-\alpha_{1}^{x}}{\alpha_{1}^{x}+\alpha_{2}^{x}} \in\right] 0,1\left[\right.$ and $\alpha_{2}^{x}=\frac{1+\beta^{x}}{1-\beta^{x}} \alpha_{1}^{x}$.

Among those $\alpha_{1}^{x}, \alpha_{2}^{x}$ satisfying this equation we can indeed choose $\alpha_{2}^{x}<\frac{1}{2}$, as we did before.

Up to now all agents in $C^{x}$ are indifferent between the original production and allocation and the new one.

Now assume that each member of $C^{x}$ instead of $\hat{x}$ even produces $\alpha^{x} N>\hat{x}$. If $\left(\alpha^{x} N_{1}-\hat{x}_{1}, 0\right)$ and $\left(0, \alpha^{x} N_{2}-\hat{x}_{2}\right)$ are distributed equally among the type 1 and type 2 agents, respectively, while $\hat{x}$ is distributed as before, all members of $C^{x}$ are better off than they were under the production of $x$. Hence $C^{x}$ improves upon $x$.

In an analogous way one can show that any $x \in \partial S$ with $x_{1}<N_{1}, x_{2}>N_{2}$ can be improved upon.

It is obvious that $N$ itself cannot be improved upon by any coalition. Also it is known from Trockel (1996) that $N$ is the unique Walrasian allocation of $\tilde{\mathcal{E}}^{S}$ and is therefore in the core of the coalition production economy $\tilde{\mathcal{E}}^{S}$ (cf. Hildenbrand (1974, p. 216). So we have established that $\{N\}=\operatorname{Core}\left(\tilde{\mathcal{E}^{S}}\right)$. 
This result could have been derived alternatively via Proposition 2 and Theorem 1 in Hildenbrand (1974, p. 216) exploiting the fact that the unique Walrasian equilibrium is the only quasi-equilibrium in $\tilde{\mathcal{E}}^{S}$ and by Trockel (1996) coincides with the Nash solution $N$ of $S$.

Our proof has the advantage to hint to the way one may get a Debreu-Scarf type convergence result for the core in our framework. This will be carried out in the next section.

\section{An Edgeworth-Debreu-Scarf approach}

In this section we are looking at the core of $n$-replicas $\mathcal{E}_{n}^{S}$ of the economy $\mathcal{E}^{S}$. Again it suffices to look at $S$. Notice that it does not make any difference whether in an $n$-replica economy every agent has the technology $Y=\frac{1}{2 n} S$ and the total production set is $S$ or wether each agent has $Y=\left(\frac{1}{2}\right) S$ and total production is $n S$. We will assume that each agent in $\mathcal{E}_{n}^{S}$ owns a production possibility set $Y:=\frac{1}{2} S$ as illustrated in Figure 3.

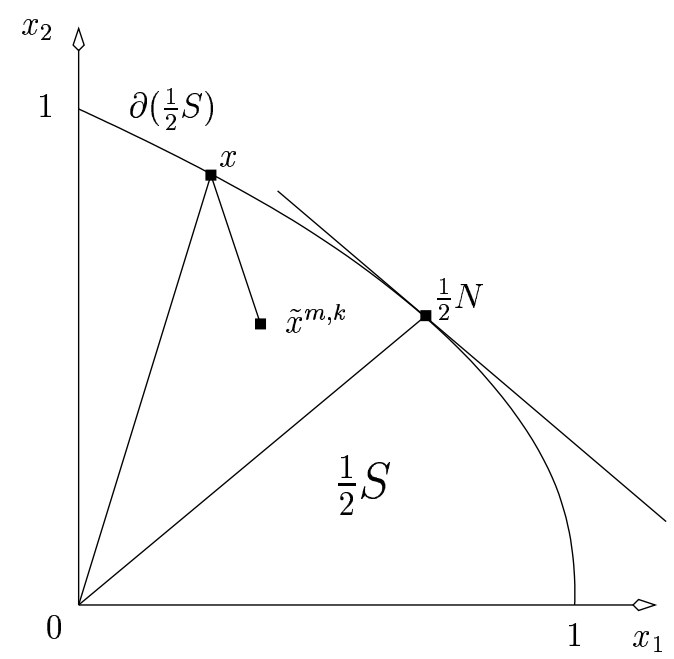

Figure 3

This time we assume w.l.o.g. that $x \in \partial\left(\frac{1}{2} S\right)$ and $x_{1}<\frac{1}{2} N_{1}, x_{2}>\frac{1}{2} N_{2}$. By choosing $n, m, k \in \mathbb{N}, k<m \leq n$ sufficiently large we can make the vector $\frac{m-k}{m+k}\left(x_{1},-x_{2}\right)$ arbitrarily small and, thereby, position the point $\tilde{x}^{m, k}:=\left(x_{1}, x_{2}\right)+\frac{m-k}{m+k}\left(x_{1},-x_{2}\right)$ in $\operatorname{int}\left(\frac{1}{2} S\right)$.

A coalition $C_{n}^{x}$ in the $n$-replica economy $\mathcal{E}_{n}^{S}$ of $\mathcal{E}^{S}$ consisting of $m$ agents of type 1 and $k$ agents of type 2 can realize the allocation $(m+k) \tilde{x}^{m, k}=$ $\left((m+k) x_{1}+(m-k) x_{1},(m+k) x_{2}-(m-k) x_{2}\right)=\left(2 m x_{1}, 2 k x_{2}\right)$. 
This bundle can be reallocated to the members of $C_{n}^{x}$ by giving to each of the $m$ type 1 agents $\left(2 x_{1}, 0\right)$ and to each of the $k$ type 2 agents $\left(0,2 x_{2}\right)$. Clearly, everybody gets thereby the same as he received in the beginning when everybody produced $x$. Therefore, nobody improves! However, for $\eta>0$ sufficiently small $\tilde{x}^{m, k} \in$ int $\frac{1}{2} S$ implies that $\tilde{x}^{m, k}+\eta N \in \operatorname{int} \frac{1}{2} S$. Now reallocation of that bundle among the members of $C_{n}^{x}$ can be performed in such a way that each type 1 agent receives $\left(2 x_{1}+\frac{m+k}{m} \eta N_{1}, 0\right)$ and each type 2 agent gets $\left(0,2 x_{2}+\frac{m+k}{k} \eta N_{2}\right)$. Therefore $x$ for every agent can be improved upon by $C_{n}^{x}$ via production of $\tilde{x}^{m, k}+\eta N$ by each of its members. Again, the only element of $\partial\left(\frac{1}{2} S\right)$ remaining in the core for all $n$-replications of $\mathcal{E}^{S}$ is the point $\frac{1}{2} N$, i.e. the Nash solution for $\frac{1}{2} S$.

Notice that any point $y \in \partial\left(\frac{1}{2} S\right)$ with $y_{1}<x_{1}<N_{1}$ can be improved upon by the same coalition $C_{n}^{x}$ via $\tilde{y}^{m, n}+\eta N$ with the same $\eta$ by a totally identical construction of $\tilde{y}^{m, n}$ from $y$.

The same is not true for $z \in \partial\left(\frac{1}{2} S\right)$ with $x_{1}<z_{1}<N_{1}$.

Here the $\frac{m-k}{m+k}\left(z_{1},-z_{2}\right)$ may require a larger $m$ and $k$ to make $\frac{m-k}{m+k}\left(z_{1},-z_{2}\right)$ small enough. We may for any $x \in \partial\left(\frac{1}{2} S\right), x_{1}<N_{1}$ choose the $m, k$ in the construction of $\tilde{x}^{m, k}$ in such a way that $\tilde{x}^{m, k}$ is on or arbitrary close to the segment $\left[0, \frac{1}{2} N\right]$. This fact will help us in the last section to drive the speed of convergence.

\section{Speed of convergence}

There are results in the literature on the speed of core convergence first by Debreu (1975) for replica exchange economies and then, more generally, by Grodal (1975) for competitive sequences of regular economies. They state that the distance between the core and the Walrasian allocations of the economies converges to zero like $1 / n$, where $n$ is the number of agents in the economy.

In our framework we get an analogous result. 


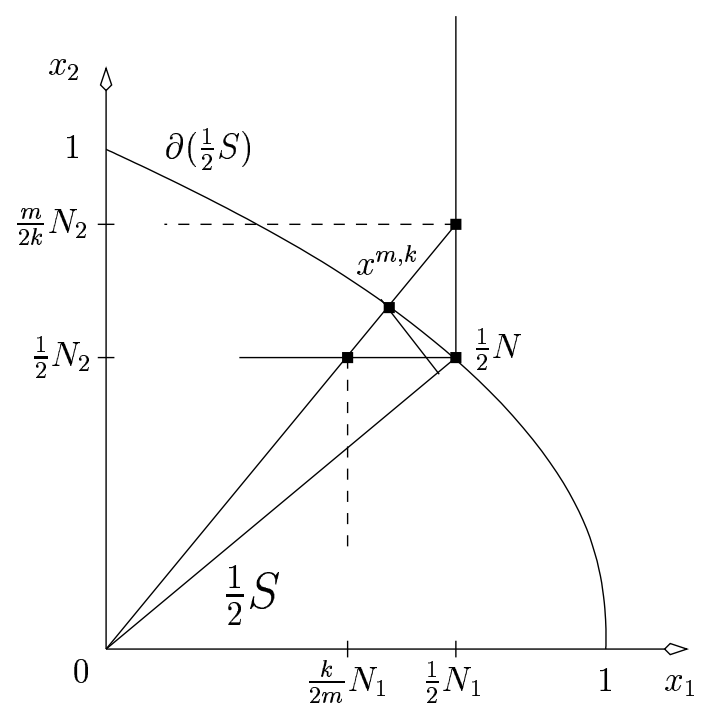

Figure 4

For given $\frac{1}{2} S$ and $\frac{1}{2} N$ we associate with any pair $k, m, 0<k<m \leq n$ the points $\frac{1}{2} N^{m, k}:=\frac{1}{2}\left(N_{1}, \frac{m}{k} N_{2}\right)$ and $\frac{1}{2} N_{m, k}:=\frac{1}{2}\left(\frac{k}{m} N_{1}, N_{2}\right)$.

Denote the unique point in the intersection

$\partial\left(\frac{1}{2} S\right) \cap\left[\frac{1}{2} N^{m, k}, \frac{1}{2} N_{m, k}\right]$ by $x^{m, k}$.

This point can be improved upon by the coalition $C_{n}^{x^{m, k}}$ via

$x^{m, k}+\frac{m-k}{m+k}\left(x_{1}^{m, k},-x_{2}^{m, k}\right)+\eta N$ for sufficiently small $\eta>0$, as we have proved in section 4 .

In fact for given $m$ of all points $x^{m, k}$ the closest one to $N$ is $x^{m, m-1}=: x^{m}$ (see Figure 4). So the length of the path on $\partial\left(\frac{1}{2} S\right)$ connecting $x^{m}$ with $N$ is an upper bound of the size of the core of $\mathcal{E}_{m}^{S}$ (or more precisely of that part of the core to the left of $\frac{1}{2} N$ ). This length is estimated from above by the number

$\max \left(\left\|\frac{1}{2} N^{m}-\frac{1}{2} N\right\|,\left\|\frac{1}{2} N_{m}-\frac{1}{2} N\right\|\right)$ with $N^{m}:=N^{m, m-1}, N_{m}:=N_{m, m-1}$.

But this number equals $\frac{1}{2} \max \left(\left(\frac{m}{m-1}-1\right) N_{2},\left(1-\frac{m-1}{m}\right) N_{1}\right) \leq \frac{1}{2} \max \left(N_{1}, N_{2}\right) \frac{1}{m-1}=0\left(\frac{1}{m}\right)$. 


\section{Concluding remarks}

The present paper continues the idea of Trockel (1996) to approach cooperative games with methods from microeconomic theory. Considering sets of feasible utility allocations as production possibility sets representing the possible jointly "producable" utility allocations and tranformation rates as prices goes back to Shapley (cf. Shapley (1969)). See also Mayberry et al. (1953). The possibility to get Core Equivalence of Walrasian equilibria of the artificial bargaining economies and to derive an Edgeworth-Debreu-Scarf type convergence result makes bargaining economies besides Edgeworth-Boxes or Robinson-Crusoe economies another attractive class of economies for illustrative purposes. The identity of the Walrasian equilibrium of a finite bargaining economy $\mathcal{E}^{S}$ with the Nash solution of its underlying bargaining game $S$ stresses the competitive feature of the Nash solution.

Moreover the Nash solution's coincidence with the Core of a large bargaining coalitional production economy with equal production possibilities for all agents reflects a different fairness aspect in addition to those represented by the axioms or by alternative characterizations. 


\section{References}

[1] Debreu, G.: The rate of convergence of the core of an economy, Journal of Mathematical Economics 2, 1-8 (1975)

[2] Debreu, G., Scarf, H.: A limit theorem on the core of an economy, International Economic Review 4, 235-246 (1963)

[3] Grodal, B.: The rate of convergence of the core for a purely competitive sequence of economies, Journal of Mathematical Economics 2, 171-186 (1975)

[4] Hildenbrand, W.: Core and equilibria of a large economy, Princeton: Princeton University Press, (1974)

[5] Mayberry, J.P., Nash, J.F. and M. Shubik: A comparison of treatments of a duopoly situation, Econometrica 21, 141-154 (1953)

[6] Nash, J.F.: Two-person cooperative games, Econometrica 21, 128-140 (1953)

[7] Shapley, L.S.: Utility comparison and the theory of games. In: La décision: aggrégation et dynamique des ordres de préférence, pp. 251-263. Paris (1969)

[8] Trockel, W.: A Walrasian approach to bargaining games, Economics Letters 51, 295-301 (1996) 